\title{
Cervicocranial Syndrome
}

National Cancer Institute

\section{Source}

National Cancer Institute. Cervicocranial Syndrome. NCI Thesaurus. Code C34411.

A neurologic syndrome following injury of the spinal sympathetic nerves of the neck. The injury usually results from arthritis or pinching by the adjacent vertebrae. Symptoms include facial pain, chronic allergies, dizziness, neck pain, ear pain and vertigo. 